Fakultät für Gesundheitswissenschaften, AG6, Versorgungsforschung/Pflegewissenschaft, Bielefeld

Doris Schaeffer (Prof. Dr.), Gabriele Müller-Mundt (Dr.)

\title{
Bewältigung komplexer Medikamentenregime bei chronischer Erkrankung - Herausforderungen und Unterstützungserfordernisse aus der Sicht der Gesundheitsprofessionen
}

- Was ist (zu dieser Thematik) schon bekannt?

Die Reformbedürftigkeit des Managements komplexer Medikamentenregime bei chronischer Krankheit.

- Was ist neu?

Der Vergleich der bislang selten untersuchten Problemsicht der am Management komplexer Medikamentenregime beteiligten Gesundheitsprofessionen. Dabei wird der ambulanten Pflege besondere Beachtung geschenkt.

- Welche Konsequenzen haben die Ergebnisse für die Pflegepraxis? Gezeigt wird, dass gerade die ambulante Pflege über zahlreiche Potenziale zur Unterstützung der Erkrankten bei einem komplexen Medikamentenregime verfügt, deren Entfaltung allerdings eine Erweiterung ihrer Kompetenzen erfordert.

zahlreiche Potenziale zur Unterstützung der Medikamentenregime bei chronischer Krankheit im Alltag besitzt, deren Ausschöpfung aber eine Erweiterung ihrer klinischen und kommunikativ-edukativen Kompetenzen voraussetzt.

Schlüsselwörter: Komplexe Medikamentenregimes, chronische Krankheit, Selbstmanagementförderung, Unterstützungserfordernisse, häusliche Pflege

Hintergrund und

Erkenntnisinteresse

Obgleich Arzneimittel zu den wichtigsten Interventionen bei chronischer Krankheit zählen, verläuft das Management des Medikamentenregimes im Alltag gerade bei älteren chronisch Erkrankten nicht optimal. Veränderungsbemühungen konzentrieren sich hierzulande meist auf die Ärzte oder Apotheker.

In der Studie, aus der hier berichtet wird, wurde der Blick auch auf die Pflege gerichtet. Gefragt wurde, wie diese drei Gesundheitsprofessionen die Problematik einschätzen, welche Unterstützungserfordernisse sie bei der Bewältigung komplexer Medikamentenregime chronisch Erkrankter sehen und welche Potenziale zur Un-

Manuskript erstmals eingereicht am 18.5.201 Endgültige Fassung eingereicht am 19.11.201 terstützung speziell der ambulanten Pflege zur Verfügung stehen. Um diese Fragen zu beantworten, wurden 26 Experteninterviews mit Vertretern der drei Gesundheitsprofessionen geführt und inhaltsanalytisch ausgewertet.

Die Ergebnisse zeigen, dass das Management komplexer Medikamentenregime von allen Experten als veränderungsbedürftig angesehen wird. Durchgängig konstatieren sie einen hohen Bedarf an kommunikativ-edukativer Unterstützung, dem bislang nicht entsprochen wird. Einhellig betonen sie zudem, dass das Management der Medikamentenregime eine multiprofessionelle Aufgabe darstellt, doch reibt sich die Umsetzung an strukturellen und professionellen Hürden. Zugleich verdeutlicht die Analyse, dass die ambulante Pflege
Die Arzneimittelversorgung ist in den letzten Jahren verstärkt in das Zentrum der gesundheitspolitischen Diskussion gerückt. Seither vergeht kaum ein Tag, an dem keine Nachricht über steigende Arzneimittelausgaben in den Medien zu finden ist und zugleich beklagt wird, dass die Arzneimittelversorgung - vor allem das Management der Medikamentenregime - be chronischer Krankheit und Multimorbidtät im Alter suboptimal ist (Glaeske, 2010; Schwabe \& Paffrath 2010; SVR, 2005). Besondere Probleme werfen komplexe Medikamentenregime auf, bei denen fünf oder mehr Präparate einzunehmen sind (Burkhardt, Wehling \& Gladisch, 2007). Sie sind gerade bei älteren chronisch Erkrankten häufig anzutreffen (Coca \& Nink, 2010; Pittrow, Krappweis \& Kirch, 2002)

Probleme des Managements der Medikamentenregime sind seit den 1970erJahren Gegenstand der Forschung zur 
Adhärenz ${ }^{1}$ (Haynes, Taylor \& Sackett, 1979; WHO, 2003) und Bewältigung chronischer Krankheit (Schaeffer, 2009; Strauss, Corbin, Fagerhaugh, Glaser, Maines, Suczek et al., 1984). Die Befunde zeigen immer wieder neu, dass die Einhaltung langfristiger und komplexer Medikamentenregime für die Erkrankten - und besonders für die älteren unter ihnen - schwierig ist: Oft erweist sich bereits die Integration des Medikamentenregimes in den Alltag für die Erkrankten schwierig. Ähnliches gilt auch für die Ausbildung von Routinen, um die Einnahme nicht zu vergessen - vor allem dann, wenn mehrere unterschiedliche Medikamente zu unterschiedlichen Zeiten eingenommen werden müssen. Probleme wirft auch das Management von unangenehmen Begleiterscheinungen, Nebenund Wechselwirkungen oder Zweifeln und Ermüdungserscheinungen auf, die im Lauf der Zeit häufig auftreten (Dybowski, 2005; Gereoni, Schönermark \& Hagen, 2007; Haynes, McDonald, Garg, Montague, 2005; Petermann, 1998; RPSGB, 1997; van Dulmen, Sluijs, van Dijk, de Ridder, Herdink \& Bensing, 2007; Vermeire, Hearnshaw, von Royen \& Denekens, 2001; WHO, 2003). Dass all diese Probleme nicht ohne gesellschaftliche Folgen bleiben, verdeutlichen die For-

1 Bewusst sprechen wir von Adhärenzforschung. Denn analog zum Wandel der Patientenrolle (McWilliam, 2009; Schaeffer, 2004; Stollberg, 2008; SVR, 2009) hat sich eine Akzentverschiebung in der «Compliance»-Debatte vollzogen Gegenüber dem mit der traditionellen ArztPatienten-Beziehung assoziierten Begriff der «Compliance» (Therapietreue) wird inwischen dem Begriff der «Adhärenz» (Therapiemotivation) der Vorzug gegeben (WHO, 2003). In jüngster Zeit wird dieser wiederum vermehrt durch den Begriff der «Konkordanz» (Übereinstimmung/Einvernehmen) abgelöst. Er steht für ein partnerschaftliches Beziehungsmodell, «in dem die Erkrankten als Person und Experten ihrer Situation unterstützt werden, informierte Ent scheidungen über die Diagnose und Therapie, über deren Nutzen und Risiken zu treffen und ein Arbeitsbündnis (২alliance) mitden professionellen Gesundheitsexperten einzugehen.» (RPSGB, 1997: 12, sinngem. Übersetzung) schungsbefunde ebenfalls: Allein die durch Adhärenzprobleme verursachten Kosten werden in Deutschland insgesamt mit jährlich schätzungsweise 10 Milliarden Euro veranschlagt (Gereoni et al., 2007: 7). Hinzu kommen Folgekosten aufgrund vermeidbarer unerwünschter Arzneimittelwirkungen. Von ihnen sind vor allem ältere Patienten ${ }^{2}$ mit einem komplexen Medikamentenregime betroffen (Thürmann, Werner, Hanke, Schmiedl, Drewelow, Hippius et al., 2007; Wu, Jen, Bottle, Molokhia, Aylin, Bell et al., 2010).

Dies deutet an, dass das Management des Medikamentenregimes bei chronischer Krankheit der Veränderung bedarf. Bei der Suche nach Wegen, wie dabei zu verfahren ist, wird im deutschen Gesundheitssystem meist ausschließlich an die Ärzte oder Apotheker gedacht (Glaeske, 2010; SVR, 2005, 2009; van Mil, Schulz \& Tromp, 2004). In der Untersuchung ${ }^{3}$, die hier im Mittelpunkt steht, wurde außerdem die ambulante Pflege in den Blick genommen. Dafür spricht nicht nur, dass hier eine multiprofessionelle Aufgabe ansteht, sondern ebenso, dass ein Großteil der mit einem komplexen Medikamentenregime für die Erkrankten einhergehenden Probleme im häuslichen Alltag manifest wird und somit für die Ärzte und Apotheker nur bedingt sichtbar ist. Die ambulante Pfle-

2 Aus Gründen der besseren Lesbarkeit wird jeweils die männliche Form für die Bezeichnung der Akteure genutzt, es sind immer auch Frauen eingeschlossen.

3 Der Beitrag basiert auf Teilergebnissen der ersten Phase des als Projekt B2 des Pflegeforschungsverbundes NRW vom Bundesministerium für Bildung und Forschung (BMBF) 05/2005 bis 03/2011 geförderten Forschungsvorhaben (BMBF-Förderkennziffer - Phase I: 01GT0315 Phase II: 01GT061; Ethikkommission der Ärztekammer Westfalen-Lippe und der Medizinischen Fakultät der Westfälischen WilhelmsUniversität Münster AZ: 2007-241-f-S). Zu dem Projektdesign und den Ergebnissen der hier im Vordergrund der Betrachtung stehenden Expertenbefragung vgl. auch den Teilbericht von Schaeffer, Müller-Mundt \& Haslbeck (2005) sowie Müller-Mundt, Haslbeck \& Schaeffer (2008) ge ist jedoch direkt im häuslichen Alltag der Patienten tätig und hat daher Einblick in die sich dort stellenden Schwierigkeiten und Interventionserfordernisse. Für den Einbezug der Pflege spricht ein weiteres Argument, denn international nimmt die Pflege im Rahmen von «Advanced Nursing Practice» zentrale Aufgaben beim Monitoring und Management des Medikamentenregimes wahr. Sie wurden in den letzten Jahren sogar um die Verordnung bestimmter Arzneimittel erweitert (Avery \& James, 2007; Cooper, Bissell, Ward, Murphy, Anderson, Avery et al., 2011; Schober \& Affara, 2008).

Inzwischen wird auch in Deutschland als überfällig angesehen, die Potenziale der ambulanten Pflege zur Optimierung der Versorgung besser auszuschöpfen (Busse, Blümel, Scheller-Kreinsen \& Zentner, 2010; Gaidys, 2011; Schaeffer, 2008; SVR, 2009) - eine Sichtweise, die sich an strukturellen und professionellen Hürden reibt (Kuhlmey, Höppner \& Schaeffer, 2011). Denn bislang sind die Interventionsmöglichkeiten der Pflege begrenzt. Die verantwortliche Mitwirkung an der Therapie und die Unterstützung der Erkrankten bei der Krankheitsbewältigung zählen zwar auch in Deutschland zu den Aufgaben professioneller Pflege ( $\$ 3, \mathrm{KrPflG}$, 2003). So gehört die Sicherstellung der medikamentösen Therapie und die Verlaufsbeobachtung im akutklinischen Bereich zu den Kernaufgaben der Pflege (Isfort, Weidner, Neuhaus, Kraus, Köster \& Gehlen, 2010: 50). Anders ist jedoch die Situation in der ambulanten Versorgung. Wird die Pflege dort auf der Grundlage der Pflegeversicherung (SGB XI) tätig, ist sie allenfalls mittelbar in das Management der Medikamentenregime eingebunden. Bei Leistungen auf Basis der Krankenversicherung (SGB V) ist sie zwar beteiligt, doch konzentriert sich ihr Part auf die Bereitstellung, Verabreichung und/oder Kontrolle der Medikamenteneinnahme. Monitoringauf- 
gaben, alltagsnahe Adhärenz- und Selbstmanagementförderung oder kommunikativ-edukative Unterstützung fallen nicht in den Aufgabenbereich der Pflege. Dies ist Resultat des verengten Pflegeverständnisses (Wingenfeld, 2011), aber auch der schwachen Position der Pflege in der Hierarchie der Gesundheitsprofessionen in Deutschland (Kuhlmey et al., 2011).

Hier Möglichkeiten der Veränderung zu untersuchen, war Ziel der Studie, aus der hier berichtet wird. Intention war, die Potenziale der ambulanten Pflege zur Unterstützung und zum Management komplexer Medikamentenregime bei chronischer Krankheit zu eruieren und nach Möglichkeiten zu suchen, ihr einen verantwortlicheren Part bei dieser als multiprofessionell angesehenen Aufgabe einzuräumen. Die Erkenntnisse sollten dazu dienen, ein bedarfsgerechtes Interventionskonzept zu entwickeln. Gefragt wurde deshalb danach, wie sich die Problematik aus der Sicht der Gesundheitsprofessionen im ambulanten Sektor darstellt, welche Herausforderungen aus ihrer Sicht für chronisch Erkrankte mit dem Management komplexer Medikamentenregime einhergehen, welche Unterstützungserfordernisse sie jeweils sehen und welche Konsequenzen daraus für die Entwicklung von Interventionskonzepten erwachsen. Dabei galt den unterschiedlichen Sichtweisen der Gesundheitsprofessionen und vor allem den in der deutschsprachigen Forschung bislang wenig beachteten Potenzialen der ambulanten Pflege besondere Beachtung.

Damit wurde eine Fragestellung aufgegriffen, die nach wie vor hochaktuell ist (Glaeske, 2010; SVR, 2009) und angesichts der epidemiologischen Entwicklung weiter an Bedeutung gewinnt. Die mit der Bewältigung der Medikamentenregime bei chronischer Erkrankung verbundenen Probleme wurden zwar - wie dargelegt - bereits seit den 1970er-Jahren untersucht. Doch haben sich in jüngerer Zeit die Kontextbedingungen in mehrfacher Hinsicht verändert: So haben sich in Deutschland die Bedingungen im Gesundheitswesen in den letzten Jahren durch gesundheitspolitische Reformen gewandelt und die Bedeutung und Position einzelner Gesundheitsprofessionen verschoben, wie unter anderem in der Stärkung der Rolle des Hausarztes und den Bemühungen zur Professionalisierung der Pflege zum Ausdruck kommt (SVR, 2005, 2007, 2009). Auch die Patientenrolle unterliegt weitgreifenden Veränderungen: In Abkehr vom benevolenten Paternalismus werden Patienten nicht mehr als passive Leistungsempfänger betrachtet, sondern als Koproduzenten ihrer Gesundheit (Donabedian, 1992). Damit verbunden haben auf Partizipation und Autonomie, Eigenkompetenz und Aktivierung setzende Versorgungsstrategien an Bedeutung gewonnen (Coulter \& Ellins, 2007; Haslbeck \& Schaeffer, 2007; Lorig \& Holman, 2003; Schaeffer \& SchmidtKaehler, 2011). Der für die Umsetzung erforderliche Kompetenzzuwachs steht jedoch noch aus und dies sowohl bei den Gesundheitsprofessionen wie auch bei den Patienten (Coulter \& Ellins, 2007; Loh, Simon, Bieber, Eich \& Härter, 2007; Müller-Mundt, 2011; Schaeffer \& Moers, 2011; SVR, 2005; 2009; WHO, 2005). Zugleich mangelt es an tragfähigen Konzepten zur Kompetenzförderung der Erkrankten, gerade auch im Umgang mit komplexen Medikamentenregimen, wie in der Adhärenz-Forschung immer wieder betont wird (Gereoni et al., 2007; Haynes et al., 2005; Ludwig, 2005; WHO, 2003).

\section{Methodik und Datenbasis}

Zur Beantwortung der Fragestellung wurde eine qualitativ angelegte mehrperspektivische Studie (Strauss, 2007) durchgeführt, die sowohl eine Exper- tenbefragung wie auch eine Befragung chronisch erkrankter Patienten umfasste (vgl. auch Haslbeck, 2007, 2010; Haslbeck \& Schaeffer, 2009; Ludwig, 2009; Müller-Mundt, Haslbeck \& Schaeffer, 2008; Schaeffer, Müller-Mundt \& Haslbeck, 2007). Der vorliegende Beitrag konzentriert sich auf die Expertenbefragung. Deren Ziel bestand darin, die Problemsicht und -einschätzung der unterschiedlichen, bei chronischer Krankheit am Management des Medikamentenregime beteiligten Gesundheitsprofessionen zu analysieren und zu vergleichen, die bislang selten Gegenstand von Untersuchungen war (Bajramovic, Emmerton \& Tett, 2004; Cooper et al., 2011). Ein weiteres Ziel bestand darin, die sich aus der Perspektive der Gesundheitsprofessionen jeweils stellenden Herausforderungen, Unterstützungserfordernisse und Optimierungspotenziale zu ermitteln.

Die Expertenbefragung basierte auf leitfadengestützten Interviews. Sie konzentrierten sich thematisch auf die jeweilige professionelle Einschätzung der Bedeutung der Arzneimitteltherapie bei chronischer Krankheit, der Probleme und Herausforderungen, die komplexe Medikamentenregime im Verlauf chronischer Krankheit und speziell im Alter aufwerfen, der Strategien der Erkrankten im Umgang mit komplexen Medikamentenregimen und des Adhärenzverhaltens im Alltag, der Rolle der Angehörigen sowie der jeweils gesehenen Unterstützungserfordernisse (siehe Tabelle 1).

Die Samplebildung zielte darauf, professionelle Akteure für die Interviews zu gewinnen, die über eine praxisgesättigte Expertise in der ambulanten Versorgung und Begleitung chronisch Erkrankter verfügen, die dauerhaft auf ein komplexes Medikamentenregime angewiesen sind. Einbezogen wurden niedergelassene Ärzte (praktische Ärzte, Allgemeinmediziner und Internisten in der hausärztlichen Versorgung), 
in öffentlichen Apotheken tätige Apotheker und Pflegekräfte ambulanter Pflegedienste (Alten- und Gesundheits-/Krankenpflege). Das primäre Auswahlkriterium war die Verfügung über Experten- und «Insiderwissen» der Handlungsbedingungen und -praxis in der ambulanten Versorgung. Die Rekrutierung der Interviewpartner aus der Ärzteschaft und der Pflege erfolgte primär über regionale professionelle Netzwerke (z.B. Hausarztnetz, regionale Pflegekonferenz). Hierzu wurden Netzwerkkoordinatoren gezielt als «Gatekeeper» kontaktiert. In die Versorgung involvierte Apotheker wurden über ein Schneeballverfahren, das heißt auf Empfehlung der Interviewpartner aus der Ärzteschaft und der Pflege rekrutiert. Insgesamt wurden 26 professionelle Akteure mit mehrjähriger Erfahrung im ambulanten Sektor in zwei Versorgungsregionen interviewt, und zwar Hausärzte, Apotheker und Pflegekräfte. Zur Arrondierung der Datenbasis und Abdichtung der Hypothesen wurden ergänzend Interviews mit ausgewiesenen Experten aus dem Bereich der unabhängigen Patientenberatung geführt. Befragt wurden:

- acht niedergelassene Ärzte (weiblich: $\mathrm{n}=3$ ), die mit durchschnittlich 19 Jah- ren über eine langjährige Erfahrung in der Primärversorgung verfügen

- fünf Apotheker (weiblich: $\mathrm{n}=2$ ) mit einer durchschnittlichen Berufserfahrung im Bereich der öffentlichen Apotheken von 13 Jahren

- zehn Pflegekräfte (weiblich: $\mathrm{n}=8$ ) aus dem Bereich der häuslichen Pflege mit einer durchschnittlichen Berufserfahrung von 11 Jahren sowie

- drei in der unabhängigen Patientenberatung tätige Sozialpädagogen und Psychologen mit langjähriger Beratungserfahrung (13 bis 20 Jahre). Die zumeist ein- bis zweistündigen Interviews (Median 90 Minuten) wurden mit dem Einverständnis der Interviewpartner aufgenommen und transkribiert. Ergänzend wurden Interviewprotokolle erstellt, in denen unter anderem Hintergrundinformationen und Spezifika der Interviewsituation und des Interviewverlaufs festgehalten wurden.

Die Auswertung der anonymisierten Interviewtexte erfolgte primär inhaltsanalytisch und orientierte sich an der themenbezogenen Analyse nach Meuser und Nagel (2005). Ergänzend zu den im Leitfaden abgesteckten Themen wurde eine am Material orientierte Entwicklung von Auswertungskategorien vorgenommen, die für die weitere Ana-

Tabelle 1: Themenschwerpunkte der Experteninterviews und der vergleichenden Analyse.

\begin{tabular}{l}
\hline Fragenkomplexe des Leitfadens \\
\hline - Bedeutung komplexer Medikamentenregime in der Versorgung \\
chronisch Erkrankter \\
- Versorgungssituation der Erkrankten \\
- Schwierigkeiten und Strategien der Bewältigung komplexer Medikamenten- \\
regime chronisch Erkrankter im Alltag \\
- Rolle des primären sozialen Netzes der Erkrankten für das Selbstmanagement \\
und Adhärenzverhalten \\
- Unterstützungserfordernisse und Defizite in der Versorgungspraxis \\
- Rolle und Potenziale der Gesundheitsprofessionen in der Versorgung \\
und Kooperationserfordernisse \\
ergänzende am Material gebildete Auswertungskategorien \\
- Akzeptanzprobleme der Chronizität der Erkrankung und der (Dauer-)Medikation \\
- Motivationskrisen im Krankheits- und Therapieverlauf \\
- Lern- und Anpassungserfordernisse der Erkrankten \\
- Oualifikations- und Kooperationserfordernisse der Gesundheitsprofessionen \\
\hline
\end{tabular}

\section{Ergebnisse: Die Problemsicht} der Gesundheitsprofessionen ${ }^{4}$

Vorab ist anzumerken, dass die Arzneimittelversorgung bei chronischer Krankheit von allen befragten Gesund-

\footnotetext{
4 Eine detaillierte Darstellung der Ergebnisse der Expertenbefragung findet sich in dem in der IPW-Veröffentlichungsreihe erschienenen Arbeitsbericht von Schaeffer, Müller-Mundt \& Haslbeck (2007). Der vorliegende Beitrag legt den Schwerpunkt die zentralen Ergebnisse der Analyse für die Entwicklung des in der zweiten Phase des Forschungsvorhabens in der ambulanten Pflege erprobten gestuften Interventionskonzept (vgl. Geuter, Müller-Mundt \& Panhorst, 2008; Müller-Mundt \& Schaeffer, 2011).
} 
Tabelle 2: Bewältigung komplexer Medikamentenregime aus Sicht der Gesundheitsprofessionen - Interventionsbedarf und -voraussetzungen.

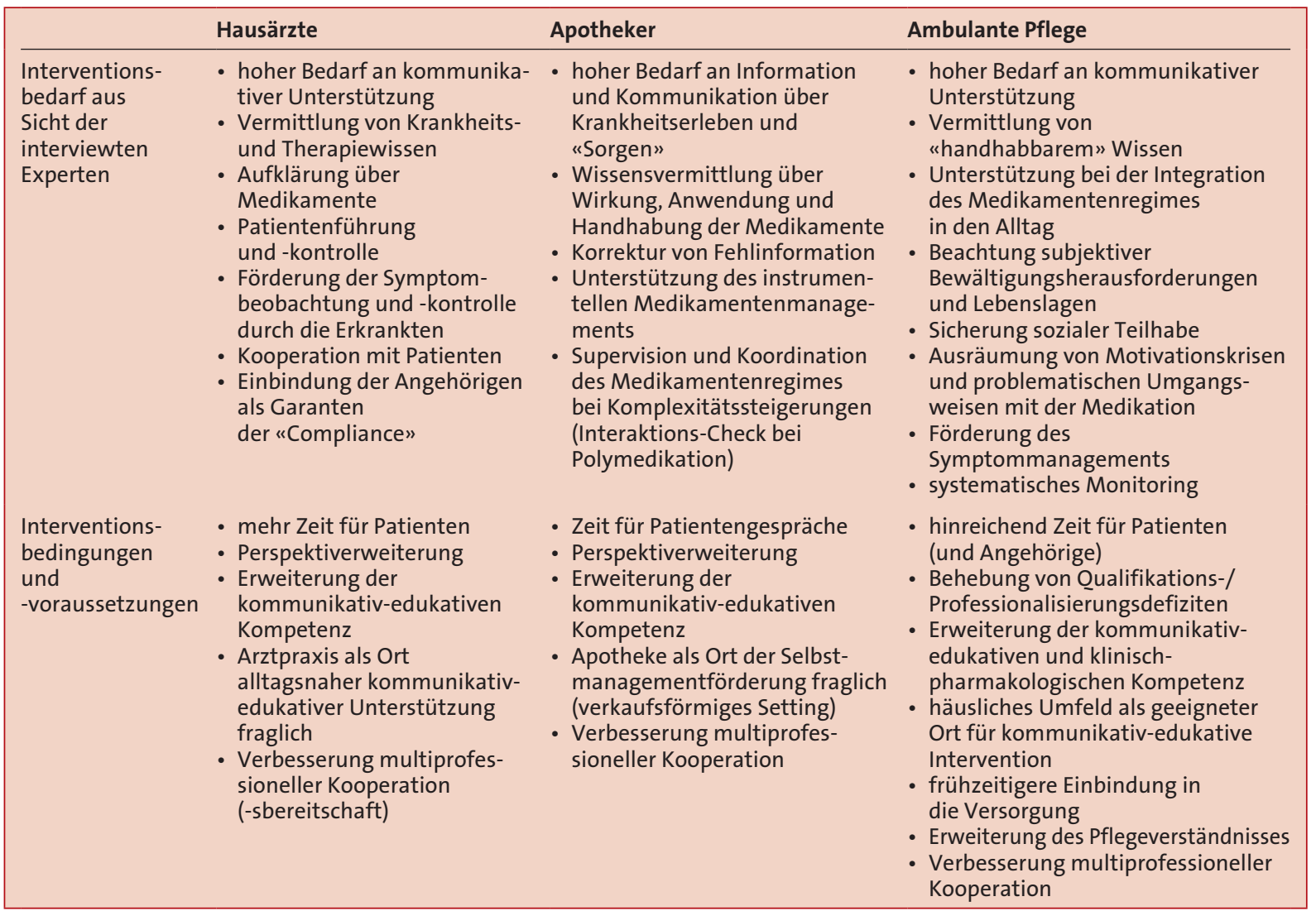

heitsprofessionen als unbefriedigend angesehen wird. Die Problemeinschätzungen sind im Detail allerdings sehr unterschiedlich und auch die Lösungsvorstellungen variieren von Profession zu Profession (siehe Tabelle 2). Um die Spezifik der jeweiligen Perspektive darstellen zu können, werden die Ergebnisse der Interviews mit den drei Gesundheitsprofessionen nacheinander abgehandelt. Dabei wird zunächst die Sichtweise der für die Arzneimitteltherapie hauptverantwortlichen Ärzte dargelegt, dann die der Apotheker und schließlich die der ambulanten Pflege.

\section{Die Sichtweise der Ärzte}

Durchgängig unterstreichen die Ärzte in den Interviews, dass sie sich als die primäre Anlauf- und Versorgungsins- tanz der Patienten begreifen - auch dann, wenn es um die Bewältigung komplexer Medikamentenregime geht. Diese ist - so die einhellige Problemeinschätzung - für die Erkrankten nicht einfach und daher fast immer mit einem hohen Bedarf an kommunikativer Unterstützung verbunden. Dass die niedergelassenen Ärzte das Potenzial dazu haben, diesen Bedarf abzudecken, ist aus ihrer Sicht keine Frage. Eher gegenteilig: Betont wird, dass sie den Kommunikationsbedarf aufgreifen müssen, um die erforderliche Mitarbeit der Patienten zu sichern. Denn sie wird als Voraussetzung für das Gelingen ihres therapeutischen Bemühens angesehen. Bedingung hierfür ist ein hinreichendes Krankheits- und Therapiewissen sowie ein Mindestmaß an
«Achtsamkeit und Selbstbeobachtung» (Arzt 02/605 f.), damit die Erkrankten ihren Gesundheitszustand einschätzen, sich anbahnende Verschlechterungen und Krisen erkennen und ihnen gegensteuern können. Denn - so wird betont: «(...) im Grunde müssen die (Patienten) mit dieser Erkrankung so leben können, dass sie eine Verschlechterung sofort irgendwie durch eine Veränderung des Therapieregimes beantworten können. Aber das (...) erfordert einen langen Atem.» (Arzt 06/124 ff.).

Diese Kompetenz kann - wie in dem Textauszug anklingt - nach Einschätzung der Ärzte nicht ohne Weiteres vorausgesetzt werden. Vielmehr setzt sie ein hohes Maß an Information und Wissen voraus, weil das Verhalten der Patienten oft rein subjektiven Rele- 
vanzkriterien folgt, die - so die Ärzte meist nicht mit medizinischen Gesichtspunkten in Übereinstimmung stehen. Betont wird, dass bei vielen chronischen Krankheiten mittlerweile eine frühzeitige medikamentöse Kontrolle für sinnvoll erachtet wird, um weitergehende Gesundheitsgefährdungen zu verhindern. Vor allem in frühen Krankheitsphasen, wenn (noch) keine gesundheitlichen Einschränkungen spürbar sind, kann die «Compliance» oft nur schwer aufrechterhalten werden. Umso wichtiger ist es den Ärzten zufolge, chronisch Kranke durch Information und Wissensvermittlung zu befähigen, «(...) die Erkrankung und auch die Wertigkeit der Medikamenteneinnahme zu verstehen.» (Arzt 01/898 ff.).

Eine große Herausforderung für die Erkrankten wird von den Ärzten darin gesehen, die Chronizität der Erkrankung zu akzeptieren. Dies gilt - wie bereits angedeutet - auch für die Dauerhaftigkeit der Medikation. Weil diese mit ihren meist an Akutkrankheiten orientierten subjektiven Krankheitsund Therapievorstellungen kollidiert, sind die Erkrankten trotz erfolgter Aufklärung durch die behandelnden Ärzte oft auf weitgehende Wiederherstellung (einst vorhandener) Gesundheit ausgerichtet. Betont wird, dass damit «Compliance»-Probleme nahezu vorprogrammiert sind: «Sich der Droge $z u$ entziehen», sobald sich eine Befindensverbesserung abzeichnet, scheint, so die Ärzte, geradezu ein «Grundbedürfnis» zu sein (Arzt 06/48 ff.). Wie der gewählte Begriff «Droge» andeutet, steht hinter dem Wunsch der Patienten nach Absetzen der Medikamente oft Furcht vor beziehungsweise der Wunsch nach Abwendung von zunehmender Abhängigkeit. Auch ihr gilt es, wie die Ärzte wiederholt betonen, durch gezielte Information und Aufklärung zu begegnen.

Das «Durchhalten» einer medikamentösen Therapie verlangt - so der
Grundtenor ihrer Aussagen - zudem ein hohes Maß an Frustrationstoleranz, besonders dann, wenn die Medikamente «unter Umständen deutliche Nebenwirkungen» haben und regelmäßige «Kontrolluntersuchungen» erfordern (Arzt 06/92 f.). Ob und inwieweit ein spürbarer Nutzen die «Compliance» fördert oder die Erkrankten eher zum Absetzen der Medikamente «verleitet», wird unterschiedlich eingeschätzt. Betont wird immer wieder, dass ein «klarer Benefit» (Arzt 03/33) sowohl zum Durchhalten der Therapie motivieren als auch Zweifel am Sinn einer weiteren Medikamenteneinnahme hervorrufen, also ungewollt kontraproduktive Effekte haben kann. Ebenso kann die Therapiemotivation nachlassen, wenn kein unmittelbarer Nutzen erlebt wird - ein Problem, das sich vor allem in frühen Krankheitsphasen stellt. In diesem Zusammenhang wird den Angehörigen eine zentrale Rolle zugesprochen. Vor allem wenn Lebensstilumstellungen erforderlich sind und sich die Erkrankten mit Komplexitätssteigerungen des Medikamentenregimes arrangieren müssen, ist es - so die Ärzte - wichtig, die Angehörigen kooperativ einzubinden. Als Dreh- und Angelpunkt aber wird von den Ärzten eine gute Patientenführung und -kontrolle erachtet. Denn oft stellt sich bereits relativ rasch «Therapiemüdigkeit» ein. Sie hat nicht selten zur Folge, dass Patienten mit den Medikamenten zu jonglieren beginnen oder sie schlicht vergessen. Die Rezeptausstellung und Kontrolluntersuchungen bieten aus Sicht der Ärzte für sie gute Anhaltspunkte für eine Kontrolle, ob die Medikamenteneinnahme auch (ver-) ordnungsgemäß erfolgt: «Etwa bei einer Hypertonie (...), wenn der (Patient) mit irgendeinem hochwertigen Antihypertonikum behandelt wird und der kommt nach sechs Wochen und hat immer noch 190 zu 100 - dann weiß ich, da stimmt irgendwas nicht» (Arzt 04/153 ff.).
Der Interviewauszug zeigt zugleich, dass die Aufmerksamkeit der Ärzte weitgehend auf objektive Krankheitsparameter gerichtet ist. Auch in der Arzt-Patienten-Kommunikation stehen - wie die Themenanalyse zeigt - offenkundig medizinische Informationen über die Erkrankung, Interventionsmöglichkeiten, Wirkungs- beziehungsweise Anwendungsweisen der Medikamente und lebensstilbezogene Verhaltensempfehlungen im Vordergrund. Wenig Raum in der Kommunikation mit den Patienten finden das subjektive Krankheits- und Therapieerleben, Probleme der Bewältigung chronischer Krankheit und der regelmäßigen Medikamenteneinnahme im Alltag ebenso wie aufkommende Zweifel, Unsicherheiten und Ängste, die Patienten mit der (Dauer-)Medikation verbinden - so ist angesichts der hierzu insgesamt recht spärlichen Aussagen der Ärzte zu vermuten.

Dass die Möglichkeiten der Patientenkommunikation im Praxisalltag begrenzt sind, wird auch von den Ärzten selbst immer wieder betont. Ursächlich dafür sind ihrer Sicht zufolge die Kommstruktur und restriktive Rahmenbedingungen der hausärztlichen Versorgung. Zwar ist die Arztpraxis aus ärztlicher Sicht der Ort der Patientenkommunikation, doch setzen dort wirksame ökonomische Zwänge und daraus resultierende enge Zeitfenster der Kommunikation deutliche Grenzen, wie in den Interviews immer wieder angemerkt wird. Sie beschränken auch die Möglichkeiten für die Herstellung partnerschaftlicher Beziehungen und die Kompetenzförderung, wie beispielhaft an der für die Krankheitskontrolle wichtigen eigenständigen Symptombeobachtung illustriert wird. Diese erfordert ein hohes Maß an Kompetenz, die zu erwerben - so die Ärzte nicht einfach ist und unerwünschte Folgen haben kann: «Sie können heute nicht 60 Leute hier haben, die Ihnen mit- 
teilen, welchen Blutdruck sie gehabt haben, vielleicht noch früh um fünf (anrufen) - sei denn, ich hab sie (dazu) aufgefordert.» (Arzt 04/947 ff.). Nicht selten ist die Selbstmessung von Krankheitsparametern - wie in dem Interviewauszug erläutert wird - kontraproduktiv und bedarf der Begrenzung, da auch im Normbereich liegende Schwankungen der Messwerte Verunsicherungen hervorrufen und einen hohen Beratungsbedarf nach sich ziehen können, der die zeitlichen Ressourcen der Arztpraxis sprengt. Wichtig ist aus Sicht der Ärzte daher, die Selbstkontrolle in angemessene Bahnen zu lenken.

Kommen wir $\mathrm{zu}$ einer Bilanzierung: Insgesamt illustrieren die Interviews eindrucksvoll, dass die Ärzte um eine aufgeschlossene Haltung und Verständnis für Compliance- beziehungsweise Adhärenz-Probleme ihrer Patienten bemüht sind. Allerdings sind die Voraussetzungen für eine adäquate Unterstützung des Managements der Medikamentenregime bei chronischer Krankheit nur bedingt gegeben. Wie das zuletzt zitierte Beispiel zeigt, sind die Ärzte nach wie vor eher traditionellen Interaktionsmustern verhaftet. Der seit längerem auch im medizinischen Diskurs proklamierte Wandel des Rollenverständnisses (von Uexküll \& Wesiak, 1990) hat die Praxis also noch nicht durchdrungen und kollidiert dort zudem mit den restriktiven Rahmenbedingungen, die wenig Raum für Kommunikation, Begleitung und Kompetenzförderung der Patienten lassen. Zugleich scheinen in den Interviews Grenzen der kommunikativen und edukativen Kompetenz auf: Zwar betonen die Ärzte immer wieder die Bedeutung der Vermittlung von Krankheitsund Therapiewissen für die Stärkung der Eigenkompetenz der Patienten. Gemessen an der Wichtigkeit und Vielfalt der sich dabei stellenden Aufgaben nehmen sich die dargelegten ärztlichen
Vermittlungsstrategien allerdings nahezu hilflos aus: Dominante Strategie ist, wie die Interviews zeigen, die mündliche Erläuterung - eine ausschließlich kognitive, auf rationale Einsichtsfähigkeit der Patienten setzende Strategie. Obschon durchaus gesehen wird, dass sie Grenzen hat und vorwiegend bei intellektuellen Patientengruppen greift, «(...) bei «einfach strukturierten〉 Menschen aber nicht.» (Arzt 04/1074 f.), halten die Ärzte an ihr fest. Doch benötigen viele Patienten andere Vermittlungsstrategien, damit sie das für sie oft sperrige und irritierende Wissen aufnehmen und sich aneignen können. Rein kognitive, auf rationale Einsichtsfähigkeit setzende Strategien zeigen dabei nur bedingt die erhofften Effekte - wie auch die Ergebnisse der Patientenbefragung unterstreichen (Haslbeck, 2007; 2010; Haslbeck \& Schaeffer, 2009; Müller-Mundt et al., 2008). Dies deutet an, wie wichtig es ist, nach neuen, auf die Patienten und ihre Situation zugeschnittenen Formen der Wissensvermittlung, Problemlösung und kommunikativen Unterstützung zu suchen, und unterstreicht zugleich, dass das Kompetenz- und Interventionsrepertoire der Ärzte ergänzungsbedürftig ist.

Es bleibt die Einschätzung der Kooperation zu betrachten, denn sie ist für eine multiprofessionelle Aufgabenwahrnehmung unverzichtbar. Auffällig ist, dass die Beziehungen zu anderen Professionen den Ausführungen der Ärzte zufolge eher lose sind. Dass die Apotheker und die ambulante Pflege einen substanziellen Beitrag zur Versorgungsgestaltung leisten (können), gerät allenfalls peripher in ihren Blick. Auch wenn hier von ärztlicher Seite «im Prinzip» Kooperationspotenziale gesehen werden, wird ihnen gegenüber eine betont distanzierte Haltung zum Ausdruck gebracht. Zudem wird die Einbindung der Expertise der Apotheker in die Versorgung als Einschrän- kung der ärztlichen Therapiefreiheit empfunden. In der ambulanten Pflege wiederum sehen die Ärzte mangels hinreichender Expertise keinen wirklichen Kooperationspartner. Sie wird - so die nahezu einhellige Einschätzung - zumeist durch wenig qualifiziertes Personal erbracht und daher eher als Notbehelf für pflegebedürftige Patienten angesehen: «Ich hab nicht das Gefühl, dass das gut abläuft, was ich da so sehe!» (Arzt 06/865 ff.) - so der Grundtenor der Aussagen.

\section{Problemwahrnehmung der Apotheker}

Auch die Apotheker betonen den hohen Bedarf an Kommunikation und Information, die komplexe Medikamentenregime mit sich bringen, und schildern diesen überdies als sehr facettenreich. Ähnliches gilt für die in der Apotheke zur Verfügung stehenden Potenziale zur Optimierung der Arzneimittelversorgung. Zwar sind auch den Apothekern zufolge die Ärzte und nicht sie die primäre Anlaufstelle für die Patienten, doch bleiben nach ihren Erfahrungen in der Arztpraxis viele Fragen unbeantwortet, wie das folgende Zitat zeigt: «Der hat mich zwar gut untersucht, mein Doktor, was ich nun aber habe, das weiß ich gar nicht und verstanden habe ich ihn auch nicht... das ging alles so schnell.» (Apotheker 02/416 ff.). Sätze wie diese sind den Apothekern zufolge häufig zu hören. Nicht selten werden beim Arztbesuch nicht angesprochene Fragen - direkt oder indirekt - daher an die Apotheker herangetragen. Ihre vorrangige Aufgabe sehen die Apotheker darin, zuzuhören: «(...) weil wir natürlich die einzigen sind, die ein bisschen Zeit für die Leute haben (...) das ist ein ganz wichtiger Punkt, diese Sozialstation Apotheke» (Apotheker 02/874 ff.). Weil sie mehr Zeit haben, werden sie oft als Ansprechpartner frequentiert und von den Patienten als Kummerkasten genutzt, bei dem sie ihre Sorgen und Nöte 
des Lebens mit Krankheit, schwierigen Lebenssituationen und Versorgungsinstanzen loswerden können. Diese Art kommunikativer Unterstützung erachten die Apotheker für überaus wichtig zur Compliance-Förderung.

Eine weitere Herausforderung und wichtige Aufgabe sehen die Apotheker darin, die Erkrankten mit der Wirkungsweise und Anwendung der Medikamente vertraut zu machen und auch Fehlinformationen auszuräumen, denn für viele Patienten sind die knappen Ausführungen oder spärlichen Notizen des Arztes nicht ausreichend oder die Äußerungen werden nicht richtig verstanden. In solchen Fällen sehen sich die Apotheker gefordert, die Dosierung und Anwendung der Medikamente zu erläutern und gegebenenfalls auch praktisch zu zeigen. Betont wird, dass die instrumentelle Handhabung der Medikamente vor allem älteren Menschen oft Schwierigkeiten bereitet. Auch die mit Mehrfacherkrankungen zumeist einhergehende Komplexitätssteigerung der Medikation ist für sie ein Problem, besonders dann, wenn eine Instanz fehlt, die das Medikamentenregime koordiniert und in Form regelmäßiger Interaktionschecks supervidiert.

Werden die Ausführungen der Apotheker unter inhaltlichen Gesichtspunkten betrachtet, fällt auf, dass Themen wie die Dosierung, die konkrete Handhabung und Einnahme der Medikamente - kurz, Aspekte des instrumentellen Medikamentenmanagements - einen hohen Stellenwert haben. Auch wenn sich die Apotheker als «Kummerkasten» verstehen, stehen die Fragen im Vordergrund, in welchem Umfang und wie Medikamente eingenommen werden sollten und weniger, wie komplexe Medikamentenregime von den Patienten bewältigt werden und welche Probleme dies in ihrem Alltag aufwirft. Dass der Apotheke eine wichtige Funktion für die Unterstützung chronisch
Erkrankter bei der Bewältigung ihres Medikamentenregimes zukommt, daran besteht aus Apothekersicht kein Zweifel. Gerade ältere Patienten verfügen meist über eine langjährig gewachsene Beziehung zu «ihrer» Apotheke, sind also sehr «apothekentreu» und haben großes Vertrauen zu den dort tätigen Personen, wie von den Apothekern immer wieder betont wird. In eigentümlichem Kontrast dazu steht, dass vor allem Aspekte der Anwendung der Medikamente in der initialen Therapiephase thematisiert werden. Auch bleibt die von den Apothekern zum Ausdruck gebrachte Wahrnehmung Probleme ihrer Patienten streckenweise sehr pauschal. In der Summe werden die Patienten - trotz Nachfrage - kaum nach Gruppen, spezifischen Verhaltensweisen und weiteren differenziert und auch nicht typisiert. Auch die Kenntnis der Lebenssituation der Patienten und der sich ihnen stellenden Herausforderungen bei der Bewältigung der Erkrankung oder des Medikamentenregimes scheint eher rudimentär zu sein. Ähnliches gilt für die (Alltags-)Routinen der Erkrankten im Umgang mit Medikamenten. Auch dazu sind die Ausführungen blass und bleiben trotz Nachfragen vage.

Ursächlich dafür dürften die strukturellen Rahmenbedingungen in der Apotheke sein. So sind die Interaktion und Kommunikation in der Apotheke durch relativ kurze und eher flüchtige Kontakte charakterisiert. Sie sind zudem stark durch das verkaufsförmige Setting geprägt, die die Kommunikation über weitergehende Fragen der Bewältigung der Erkrankung und des Medikamentenregimes begrenzt. Verstärkt wird dies durch die geschäftsförmigen Räume und den öffentlichen Charakter der Apotheken, der zumeist die Anwesenheit unbekannter Dritter impliziert. Dies behindert eine vertrauliche Kommunikation und den Austausch über intime Belange, die aus
Sicht der Patienten die Privatsphäre tangieren, also der Öffentlichkeit nicht oder nur unter bestimmten Bedingungen preisgegeben werden.

Hinzu kommt, dass die Mehrheit der Patienten - wie in den Interviews betont wird - an einem traditionellen Bild von der Apotheke festhält und diese in erster Linie als «Abgabestelle von Medikamenten» (Apotheker 02/21) ansieht, aber nicht «unbedingt in der Apotheke eine umfassende Beratung (erwarten). Eigentlich ist es für einen Großteil immer noch so, die wollen möglichst schnell ihr Medikament abholen und dann fertig!» (Apotheker 01/794 ff.). Auch dieses Verhalten dürfte dem verkaufsförmigen Setting geschuldet sein, das die Erkrankten offenkundig nicht dazu anregt, länger dauernden Erklärungen zuzuhören: «Und wenn man gerade das Herz erklärt und will übergehen zu den Medikamenten, ... dann haben die keine Zeit mehr und gehen!» (Apotheker 03/581 ff.), so die ernüchternde Einschätzung, die sich durch fast alle Interviews zieht. In der Gesamtschau deutet dies an, wie wichtig es ist, auch in der Apotheke die für Information und Beratung erforderlichen Voraussetzungen zu schaffen. Neben zeitlichen Ressourcen zählt hierzu die Herstellung einer weniger verkaufsförmigen Atmosphäre, einschließlich geeigneter Bedingungen für eine Beratung im geschützten Raum. Ebenso wichtig ist eine Erweiterung der Perspektive und Beratungskompetenz, denn auch die Apotheker tendieren dazu - wie die Interviews eindrucksvoll belegen - ihre kommunikative Kompetenz zu überschätzen. Zwar werden psychosoziale und emotionale Unterstützungserfordernisse der Erkrankten gesehen. Vornehmlich aber sind die Apotheker auf die Erklärung von Fragen rund um die Tablette konzentriert und setzen dabei wie die Ärzte auf kognitive Strategien der Informations- und Wissensvermittlung und auf rationale 
Einsichtsfähigkeit der Patienten. Dadurch gelingt ihnen kaum, die meist verdeckt in Alltagserzählungen artikulierten Probleme und Unterstützungsbedürfnisse der Patienten zu dekodieren und Informations- und Beratungsangebote so zuzuschneiden und $\mathrm{zu}$ platzieren, dass sie auf Akzeptanz und Gehör stoßen. Dies zeigt, wie wichtig es ist, auch die kommunikative Kompetenz der Apotheker zu verbessern.

Betrachten wir abschließend, wie die Apotheker die für eine multiprofessionelle Versorgung erforderliche Kooperation einschätzen. Einhellig wird deren Bedeutung unterstrichen, doch zugleich betont, dass sich die Zusammenarbeit mit der Pflege meist auf die Bereitstellung der Medikamente beschränkt und sie von den Ärzten nicht als professionelle Kooperationspartner im Versorgungsgeschehen akzeptiert werden. Eher gegenteilig: selbst bei komplexen Medikamentenregimen ist es für sie schwierig, mit Hinweisen zu möglichen Unverträglichkeiten und Wechselwirkungen zum Arzt durchzudringen. Entsprechende Aktivitäten, so der Grundtenor der Aussagen, werden oft als Übergriff in die ärztliche (Be-) Handlungsautonomie abgewehrt.

Die Perspektive der ambulanten Pflege Auch von der ambulanten Pflege wird - ähnlich wie von den Ärzten und Apothekern - der hohe Unterstützungsbedarf chronisch Erkrankter bei dem Management komplexer Medikamentenregime betont und dabei auf Dimensionen aufmerksam gemacht, die sich partiell mit denen der Ärzte und Apotheker decken, partiell jedoch unterscheiden. Zwar heben auch die Pflegenden die Bedeutung von Krankheits- und Medikamentenwissen hervor: «Der Mensch, der das schlucken soll, muss wissen warum!» (Pflege 04/259 f.), so der Grundtenor der Aussagen. Zugleich wird von ihnen aber immer wieder unterstrichen, dass die
Vermittlung von abstraktem Wissen nicht ausreicht, sondern die Erkrankten Verständnis und handhabbares Wissen benötigen, das ihrer Situation Rechnung trägt: «(...) dann würden die Patienten auch viel besser ihre Medikamente einnehmen, weil sie einfach wissen, wofür ist das denn, warum muss ich das nehmen, wie lang muss ich das nehmen, (...) kann ich das mal wieder absetzen oder muss ich das mein Leben lang nehmen» (Pflege 10/1916 ff.).

Auch der technisch-instrumentelle Unterstützungsbedarf im Umgang mit der Medikation ist aus Sicht der Pflege nicht durch bloße Information und Demonstration der Handhabung hinreichend beantwortet, sondern eingebettet in den Lebensalltag und seine Routinen anzugehen. Exemplarisch zeigt dies, was wesentliches Merkmal des Problemzugriffs der ambulanten Pflege ist: Alltagsnähe ist für sie typisch. Wie der nachfolgende Textauszug illustriert, sieht sie sich daher auch in einer Mittlerfunktion: «Wir sind ja eigentlich so das Verbindungsglied (...) zwischen dem Patient und dem Arzt (...), die (Ärzte) haben den Patienten wochenlang, monatelang nicht gesehen, wir sind aber täglich vor Ort und das meistens über mehrere Wochen kontinuierlich hinweg, (...) und (können) Patienten über mehrere Wochen beobachten. Und wir haben ja eine ganz andere Beobachtungsfähigkeit (...) als der Arzt.» (Pflege 03/581 ff.).

Die alltagsgesättigte Zugriffsweise ist Resultat dessen, dass die Pflege vor Ort tätig ist und vor allem für chronisch Erkrankte, die sich nicht mehr autonom versorgen können, eine wichtige Instanz darstellt. Dies erklärt, warum sie - wie die Interviews illustrieren - die Herausforderungen, die ein komplexes Medikamentenregime mit sich bringt, sehr alltagsgesättigt $\mathrm{zu}$ schildern vermag. Typisch für die Sichtweise der Pflege ist zudem, dass sie den mit chronischer Krankheit einhergehenden Begrenzun- gen und emotionalen Bewältigungsprobleme besondere Beachtung schenkt. Auch die Pflegenden betonen die Bedeutung des Arztes für die Patienten. Zugleich weisen sie auf zeitliche und kommunikative Grenzen der Ärzte: «Die haben einfach nicht genug Zeit oder finden auch nicht die richtigen Worte, das zu erklären» (Pflege 04/787 f.) - so der Grundtenor der Aussagen der Pflegenden. Häufig sieht sich daher auch die Pflege vor die Aufgabe gestellt, Patienten offen gebliebene Fragen zu erklären. Damit allein ist es jedoch nicht getan: Um «Compliance» zu ermöglichen, bedarf es ihrer Einschätzung nach außerdem der Vermittlung von Krankheits- und Therapiewissen und der Unterstützung dabei, das Medikamentenregime in den Alltag zu integrieren. Dabei sind - so wird einhellig betont - gerade bei älteren Menschen im fortgeschrittenen Krankheitsstadium die Lebensumstände zu beachten: «Es ist auf jeden Fall auch für einen Arzt nicht unwichtig zu wissen, wie die Lebensumstände dieses Menschen sind, dem er die Medikamente verschreibt.» (Pflege 04/802 ff.). Vielen Ärzten wird von der Pflege jedoch eine gewisse Gedankenlosigkeit attestiert, weil sie zu wenig reflektieren, ob die Patienten etwa angesichts zunehmender altersassoziierter Funktionseinbußen (noch) in der Lage sind, das Medikamentenregime zu verstehen und einzuhalten.

Deshalb ist aus Sicht der Pflege essenziell, sich zu vergewissern, ob Anwendungshinweise verstanden wurden und angemessen umgesetzt werden können. Nicht selten sehen sich die Pflegenden vor die Aufgabe gestellt, die eher kargen Informationen des Arztes $\mathrm{zu}$ ergänzen und in eine für die $\mathrm{Pa}-$ tienten verständliche Sprache zu übersetzen, die für eine sachgerechte Handhabung der Medikamente nötigen Kompetenzen $\mathrm{zu}$ vermitteln und zudem nach Wegen zu suchen, das Me- 
dikamentenregime in den Alltag zu integrieren und dort stützende Routinen auszubilden: «Man muss einfach mit demjenigen zusammen überlegen, wie er am besten seine Medikamente einnehmen kann und wie er auch selber dran denken kann» (Pflege 04/274 ff.). Dabei spielt, wie in dem Zitat zum Ausdruck gebracht wird, die Entwicklung von Strategien «wider das Vergessen» der Medikamenteneinnahme eine zentrale Rolle.

Eine weitere zentrale Aufgabe besteht aus Pflegesicht darin, den Erkrankten trotz Krankheit und eines einschränkenden Medikamentenregimes soziale Teilhabe zu ermöglichen. Dazu gehört etwa abzuklären, welcher Spielraum bei der Einnahme besteht, ohne die therapeutische Wirkung zu beeinträchtigen. Als Beispiel wird hier zumeist auf die flexible Handhabung der Diuretika-Einnahme verwiesen, um «unbeschwert» an geselligen Aktivitäten teilnehmen zu können. Weil dies langfristige «Adhärenz» fördern kann, ist aus Sicht der Pflege wichtig, in Abwägung der Lebensqualität bewusst und konstruktiv mit «Non-Compliance» umzugehen.

Eine nicht zu unterschätzende Herausforderung stellen auch aus Sicht der Pflege Akzeptanzprobleme dar, denn vor allem bei dauerhafter Angewiesenheit auf Medikamente entwickeln Patienten im Lauf der Zeit oft Motivationskrisen und teilweise «regelrechte Aversionen gegen die Medikamenteneinnahme». Diese zu überwinden erfordert harte und langwierige professionelle Arbeit: «Dass den Menschen klar ist, dass die Dauermedikation (...) auf Dauer mehr (hilft), also das ist ein langer Weg.» (Pflege 04/912 ff.) - so der Grundtenor der Aussagen der Pflegenden. Zudem ist immer wieder zu beobachten, dass sich Zweifel am Nutzen der Medikamente einstellen und die Erkrankten keinen Sinn mehr in der Therapie sehen - seien diese Motivati- onskrisen durch Enttäuschung darüber verursacht, dass die Krankheit nicht zum Stillstand kommt, oder «(...) in Verbindung mit Depressionen, dass sie dann sagen, ich will gar nichts mehr nehmen» (Pflege 08/188 ff.).

Vor allem ältere und schwerkranke Patienten sind - so die Pflege - meist dann «compliant», wenn sie «noch irgendein Ziel» vor Augen haben, für das es sich lohnt, das Medikamentenregime einzuhalten (Pflege 01/1475 f.). Gerade bei ihnen ist daher aus Sicht der Pflege wichtig, Motivationskrisen und Therapieüberdruss frühzeitig zu erkennen und zu versuchen, durch kommunikative Begleitung einen Therapieabbruch $\mathrm{zu}$ vermeiden. Exemplarisch wird hier die hohe Bedeutung sichtbar, die psychosozialen Aspekten von der Pflege beigemessen wird. Dies gilt ebenso für die Einbindung der Angehörigen.

Für ähnlich wichtig werden eine begleitende Unterstützung sowie ein systematisches Monitoring gehalten. Sie sind - so die Pflege - vor allem bei älteren Patienten erforderlich, deren Fähigkeiten zum eigenständigen Symptom- und Medikamentenmanagement eingeschränkt sind. Betont wird, dass Angehörige zwar oft bemüht sind, dies zu kompensieren, doch benötigen sie eine angemessene «Schulung», denn auf der Basis «naturwüchsiger» Kompetenzen ist Aufgaben wie Symptombeobachtung und -deutung nur bedingt Gelingen beschert: «Wie erkennt man denn Nebenwirkungen? Dazu müssen Sie ja wissen, was steht da im Beipackzettel (...). Und natürlich auch eine gute Beobachtungsgabe haben. Aber wer kann das denn? Angehörige? Wenn man sie schult, sicherlich. Aber man schult sie ja nicht.» (Pflege 01/125 ff.). Da es an solchen Anleitungen mangelt, übernimmt die Pflege diese Aufgabe oft «nebenbei», erachtet aber für dringend nötig, ihr mehr Raum zuzubilligen.

Besondere Herausforderungen ergeben sich auch aus Sicht der Pflege, wenn die Komplexität des Medikamentenregimes zunimmt: Vor allem bei älteren Menschen gleichen die Medikamentenregime im Lauf der Zeit einem «Sammelsurium» (...), das (sie) dann irgendwie selber regeln sollen.» (Pflege 01/68). Weil Hausbesuche der Ärzte rar sind, entziehen sich solche Probleme dem ärztlichen Blick. Sie werden - da oft schambesetzt - zudem von Patientenseite kaum zur Sprache gebracht. Ähnliches gilt aus Sicht der Pflege für Schwierigkeiten der Umsetzung des Medikamentenregimes im Alltag, das Jonglieren mit Medikamenten und auch für das Thema Selbstmedikation, sei es durch freiverkäufliche Medikamente oder Rückgriff auf Restbestände abgesetzter Medikamente: «Ganze Apotheken sind da im Schrank (...), das weiß auch der Hausarzt nicht.» (Pflege 10/520 ff.).

Auch aus diesen Gründen wird ein systematisches Monitoring für sinnvoll erachtet. Dass dies eine wichtige Pflegeaufgabe ist, steht für die Pflege außer Frage. Dabei wird auf die aus der $\mathrm{Zu}-$ gehstruktur und Alltagsnähe erwachsenen Unterstützungspotenziale verwiesen, ebenso darauf, dass die ambulante Pflege vergleichsweise engmaschig im häuslichen Umfeld präsent ist und daher Einblick in Anpassungsschwierigkeiten und das soziale beziehungsweise familiale Umfeld hat, so auch in die sich dort stellenden psychosozialen Herausforderungen und Schwierigkeiten: «Man geht bei jedem Patienten in sein (...) ganz intimes Umfeld und je länger man einen Patienten kennt und je vertrauter man sich wird, (...) ist (man) irgendwann nicht nur noch Pflegepersonal, man ist (...) fast eine Art Angehöriger.» (Pflege 03/2653 ff.). Zudem steht die Pflege - wie in dem Zitat exemplarisch zum Ausdruck kommt - in einer durch «Nähe» und «Vertrautheit» charakterisierten Beziehung zu den Patienten, die es ihr ermöglicht, alltägliche Probleme mit 
der Medikation anzugehen und schambesetzte Themen anzusprechen. Der Ausschöpfung der Potenziale der Pflege stehen allerdings ebenfalls strukturelle Hindernisse entgegen, allem voran finanzielle und zeitliche Restriktionen. «Medikamentengabe (...) wird ja für uns nicht richtig abgerechnet, (...) wir kriegen fünf Minuten Zeit dafür.» (Pflege 10/2039 ff.) - so die Kommentare zur Vergütung von SGB V-Leistungen zur Sicherstellung der medikamentösen Therapie.

In der Summe zeigt sich, dass in der ambulanten Pflege zahlreiche Potenziale für eine alltagsnahe Unterstützung des Managements der Medikamentenregime der Erkrankten und/oder ihrer Angehörigen angelegt sind. Doch erweisen sich die Interventionsvoraussetzungen ebenfalls als strukturell begrenzt. Wie in dem zuletzt zitierten Textauszug anklingt, kann kommunikativ-edukative Unterstützung unter den gegebenen Bedingungen in der ambulanten Pflege nur finanziell ungedeckt erbracht werden. Zudem kommen Professionalisierungs- und Qualifizierungsdefizite zum Tragen. So zeigt die Analyse, dass die Pflege zwar über ein großes Verständnis für die Patientenprobleme und ein breites Alltagsund Erfahrungswissen verfügt, doch vermag sie ihr «implizites» Wissen nicht hinreichend in reflektiertes «explizites» Wissen zu übersetzen. Darüber hinaus ist die für ein qualifiziertes Monitoring erforderliche pharmakologische Expertise ergänzungsbedürftig und dies gilt auch für die kommunikativ-edukativen Kompetenzen.

Deutlich wird in der Gesamtschau, dass sich die Pflege zwar durch große Sensibilität für die psychosozialen und lebensweltlichen Probleme im Umgang mit chronischer Krankheit und der Arzneimitteltherapie auszeichnet, ebenso für Verlust- und Leidenserfahrungen. Emphatische Anteilnahme und Sensibilität reichen jedoch nicht aus, um den kommunikativ-edukativen Unterstützungsbedarf der Patienten zu beantworten. Vielmehr ist erforderlich, die Pflege in die Lage zu versetzen, ihre «intime» Kenntnis der Patientenprobleme für eine systematische Kompetenzförderung nutzbar zu machen. $\mathrm{Zu}-$ gleich zeigen sich auch in der Pflege strukturelle Grenzen: Allem anderen voran sind hier das verengte Pflegeverständnis und die späte Hinzuziehung der ambulanten Pflege zu erwähnen Statt frühzeitig als supervidierende Instanz in die Versorgung einbezogen zu werden, wird sie meist erst in Spätphasen chronischer Krankheit hinzugezogen. Dies erlaubt ihr nur, auf Motivationseinbrüche und die Arzneimittelwirkung beeinträchtigende Verhaltensmuster «zu reagieren» $(\mathrm{PF}$ 02/1078 ff.), nicht aber vorausschauend und präventiv zu agieren. Damit werden viele ihrer Potenziale verschenkt. Abschließend bleibt, die Kooperation zu betrachten: Deren hohe Bedeutung wird auch von der Pflege immer wieder unterstrichen und gleichzeitig auf Hürden aufmerksam gemacht: Während sich die eher pragmatische Kooperation mit den Apotheken als problemlos darstellt, reibt sich die Zusammenarbeit mit der Medizin an zahlreichen, aus Pflegesicht nahezu unlösbar erscheinenden Problemen. Daher wird zwar konzediert, dass beide Professionen theoretisch über viele Möglichkeiten zur Optimierung des Managements komplexer Medikamentenregime verfügen, doch dominieren seitens der Pflege Zweifel, ob diese angesichts der bestehenden Macht-, Status- und Akzeptanzschwierigkeiten zwischen Medizin und Pflege ausschöpfbar sind.

\section{Diskussion und Schlussfolgerungen}

Versuchen wir an dieser Stelle die Ergebnisse kontrastierend zusammenzu- führen. Wie die zurückliegenden Ausführungen gezeigt haben, wird das Management komplexer Medikamentenregime bei chronischer Krankheit von allen befragten Professionen als Herausforderung bezeichnet, die im hiesigen Gesundheitswesen noch keineswegs befriedigend gelöst ist. Auch darüber, dass die hier bestehenden Probleme dringend anzugehen sind und es dazu neuer Konzepte bedarf, herrscht Einigkeit. Unterschiede zeigen sich allerdings, wenn es um die Frage geht, wer die dazu geeignete Instanz ist. Dann reklamiert jede der befragten Gesundheitsprofessionen für sich, die «eigentlich richtige» Unterstützungsinstanz zu sein. Dass hier eine multiprofessionelle Aufgabe zur Bewältigung ansteht, ist noch wenig im Bewusstsein der Gesundheitsprofessionen verankert und kollidiert mit Vorbehalten gegenüber den anderen Gesundheitsprofessionen - besonders seitens der Ärzte -, ebenso mit bestehenden Kooperationsmustern. Insgesamt, so zeigen die Ergebnisse der Analyse, ist die professionsübergreifende Kooperation in der ambulanten Versorgung sehr störanfällig und reibt sich nach wie vor an der bestehenden Hierarchie und dem Machtgefälle der Professionen sowie an Akzeptanzproblemen (Garms-Homolová \& Schaeffer 1998; Shaw, De Lusignan \& Rowlands, 2005). Hier eine Veränderung herbeizuführen und nach neuen nicht-hierarchischen, sondern aufgabenorientierten Wegen der Zusammenarbeit zu suchen, ist eine unabdingbare Voraussetzung für eine multiprofessionelle Arzneimittelversorgung bei chronischer Krankheit.

Dass der ambulanten Pflege bei der Bewältigung komplexer Medikamentenregime eine bedeutsame Rolle zukommenkönnte, dieüberdieWahrnehmung reiner Kontrollfunktionen und arztentlastender Aufgaben hinausgeht (SVR, 2007), ist - wie die zurückliegenden 
Ausführungen, aber auch die jüngsten Reformdebatten zeigen - den Ärzten und Apothekern relativ fremd. Ohne nicht auch hier und im Verhältnis der Professionen zueinander eine Veränderung herbeizuführen, dürfte es schwierig sein, der Pflege eine relevante Rolle bei der Unterstützung der Probleme, die das Management des Medikamentenregimes im Alltag aufwirft, zuzuweisen. Doch hat die zurückliegende Analyse bestätigt, dass der ambulanten Pflege dafür zahlreiche Potenziale zur Verfügung stehen. $\mathrm{Zu}$ erwähnen ist nochmals die alltagsgesättigte $\mathrm{Zu}$ griffsweise, ihre relativ engmaschige Anwesenheit im häuslichen Umfeld der Erkrankten, die damit einhergehende Nähe zu den Erkrankten, ebenso ihre Sensibilität für die psychosoziale Situation und die Bewältigungsprobleme der Erkrankten. Jedoch wurden auch bei der ambulanten Pflege Probleme sichtbar, die sich als Hemmnis erweisen. Dazu gehören Professionalisierungs- und Qualifikationsdefizite ebenso wie die der deutschen Pflege attestierte Selbstbescheidenheit (Döhler, 1997) und Zurückhaltung, wenn es darum geht, eigeninitiativ einen verantwortlichen Part $\mathrm{zu}$ übernehmen (di Luzio, 2008). Ohne nicht auch dies zu verändern und $\mathrm{zu}$ einem neuen Rollenverständnis zu gelangen, dürfte kaum möglich sein, die Pflege zur wichtigen Unterstützungsinstanz bei dem Management komplexer Medikamentenregime zu erheben.

Übereinstimmend wird von allen befragten Gesundheitsprofessionen der große Bedarf an kommunikativ-edukativer Unterstützung der Erkrankten bei der Bewältigung komplexer Medikamentenregime betont. Die Vermittlung von hinreichendem Krankheits- und Medikamentenwissen wird durchgängig als unverzichtbar für die Ermöglichung von «Compliance» erachtet. Die Akzentsetzungen sind, wie zu sehen war, unterschiedlich und reichen von der Betonung rein kognitiven Wissens über instrumentelle Fertigkeiten bis hin zur Kompetenz- und Selbstmanagementförderung, um das Medikamentenregime im Alltag bewältigen zu können. Auch wenn also Konsens über die Wichtigkeit kommunikativ-edukativer Unterstützung besteht, variieren die jeweils gesehenen Lösungsvorstellungen. Sie sind zugleich stark durch die unterschiedlichen professionellen Zugriffsweisen geprägt. Trotz dieser Unterschiede wurde aber auch deutlich, dass alle befragten Gesundheitsprofessionen eine Kompetenzerweiterung benötigen, damit sie in die Lage versetzt werden, den konzedierten kommunikativ-edukativen Unterstützungsbedarf befriedigend aufgreifen zu können. Wie in der Literatur betont wird, sind kommunikative Unterstützung, Patientenedukation und -beratung sowie Selbstmanagementförderung keine Aufgaben, die auf naturwüchsiger Basis wahrgenommen werden können. Vielmehr erfordern sie spezielle Kompetenzen (Koerfer, Köhle \& Obliers, 1994; Müller-Mundt, 2011; Pols, Battersby, Regan-Smith, Markwick, Lawrence, Auret et al., 2009; Kuhlmey et al. 2011; Schaeffer \& Dewe, 2006; Shaw et al., 2005), die trotz diverser Reformen noch nicht in der erforderlichen Breite und Tiefe Gegenstand der Ausbildung der Gesundheitsprofessionen sind und daher eine zusätzliche Qualifizierung bedürfen. Dies gilt, wie die Analyse zeigt, besonders, aber nicht allein für die Pflege.

Durchgängig - so ein weiterer Aspekt zeigen die befragten Gesundheitsprofessionen zwar Verständnis für Patienten- und Compliance-Probleme. Doch trotz der seit längerem geführten Diskussion über den Wandel der Patientenrolle (Kranich, 2004; McWilliam, 2009; Schaeffer, 2004; Stollberg, 2008; SVR, 2009) scheint der Versorgungsalltag weiterhin eher durch herkömmliche Rollen- und Interaktionsmuster geprägt zu sein. Dies gilt besonders für die Ärzte. Sie sind noch weitgehend einem traditionellen Rollenverständnis verhaftet, das die Sicht auf die Probleme, die der Alltag mit chronischer Erkrankung und komplexen Medikamentenregimen für die Erkrankten aufwirft, versperrt. Das bestätigt sich auch, wenn ihre Ausführungen unter inhaltlichen Gesichtspunkten betrachtet werden. Dann wird sichtbar, dass sie vorwiegend aus der Expertensicht argumentieren und es ihnen schwer fällt, bei der Suche nach Lösungsansätzen und Optimierungspotenzialen von den Patienten her zu denken und damit den für eine bedarfs- und bedürfnisgerechte Versorgung erforderlichen Perspektivwechsel vorzunehmen. Diese Schwierigkeiten sind bei der Pflege geringer ausgeprägt. Dennoch wurde insgesamt sichtbar, dass die Realisierung von Patientenorientierung und Bedarfsgerechtigkeit insgesamt noch vor Herausforderungen gestellt ist.

Die Analyse hat außerdem darauf aufmerksam gemacht, dass die Problemwahrnehmung und -einschätzung aller drei Gesundheitsprofessionen bei näherer Betrachtung als begrenzt einzuschätzen ist. Teilweise lässt sie sich als somatisch beziehungsweise pragmatisch verkürzt oder aber als technischinstrumentell verengt charakterisieren, so bei den Ärzten oder den Apothekern. Teilweise wiederum leidet sie unter einem Mangel an systematischem Wissen. Dies gilt speziell für die Pflege - ein Befund, der angesichts der Qualifikationssituation in der Pflege nicht erstaunt (Kuhlmey et al., 2011; WHO, 2005; Winter, 2008). So wurde sichtbar, dass es ihr vor allem an hinreichenden klinisch-pharmakologischen Kompetenzen, speziell der geriatrischen Pharmakologie mangelt. Doch nicht nur bei der Pflege, auch bei den anderen befragten Gesundheitsprofessionen sind, so wurde deutlich, Qualifizierungsmaßnahmen erforderlich, um 
zu einer Perspektiverweiterung zu gelangen und ein umfassendes Management komplexer Medikamentenregime bei chronisch Erkrankten zu ermöglichen.

Darüber hinaus sind wissenschaftlich fundierte Interventionskonzepte gefordert, denn bislang agieren alle drei Gesundheitsprofessionen vorrangig auf der Basis von Erfahrungswissen - eine nur bedingt tragfähige Strategie, wie auch in der Compliance-und Adhärenzdebatte seit langem betont wird. Ebenso wird betont, dass auf der Ebene der Konzeptentwicklung noch viel Entwicklungsarbeit erforderlich ist (Chodosh, Morton, Mojica, Maglione, Suttorp, Hilton et al., 2005; Coulter \& Ellins, 2007; de Silva 2010; Gaidys, 2011; Gereoni et al., 2007; Greenhalgh, 2009; Haslbeck \& Schaeffer, 2007; Loh et al., 2007; Müller-Mundt, 2011; Robinson, Callister, Berry \& Dearing, 2008; WHO, 2005). Die zurückliegende Analyse dürfte für die Erarbeitung solcher Konzepte wertvolle Hinweise liefern, ebenso für die Reflektion geeigneter Rahmen- und Interventionsbedingungen. Sie sind, wie zu sehen war, in allen hier thematisierten Settings noch unbefriedigend. Auch für die Erarbeitung von Interventionskonzepten für eine von der ambulanten Pflege getragene Unterstützung des Umgangs mit dem Medikamentenregime liefert sie zahlreiche Anregungen.

\section{Danksagung}

Unser besonderer Dank gilt den professionellen Akteuren, die sich in der ersten Projektphase für ein Experteninterview zur Verfügung gestellt haben, sowie Jörg Haslbeck und Sandra Jansen für die Durchführung der Experteninterviews.
Management of complex medication regimes in chronic illness - challenges and support needs from the health professional's view

While chronic illness are mostly treated with pharmaceutical means, the management of medication regimes in everyday life often remains inadequate, especially for elderly people. In Germany, most efforts to change this situation focus on the role of physicians or pharmacists respectively. In contrast, this study concentrates on home care nurses and posits their potential to improve the management of complex medication regimes. To explore the professional's view 26 expert interviews with representatives of the different healthcare professions were conducted and analysed.

The results indicate that regardless of their profession, all interviewees see a need to modify existing medication regimes and share the view that there is a necessity of communicative and educational support of patients. They also agree that improvements in the management of medication require a multi-professional approach and that home care nurses could provide substantial support to chronically ill in managing their daily medication regimes. Nevertheless, the experts also report structural and professional barriers to hinder professionals in meeting these demands. We conclude that an enhancement of nurses' clinical and educational skills is inevitable, if they are to support chronically ill in managing their daily medication regimes in cooperation with other professions.
Key words: multiple medication regimes, chronic illness, support needs, home care

Literatur

Avery, A.; James. V. (2007). Developing nurse prescribing in the UK. British Medical Journal, 335, 315-316.

Bajramovic, J.; Emmerton, L.; Tett, S. E. (2004). Perceptions around concordance - focus groups and semistructured interviews conducted with consumers, pharmacists and general practitioners. Health Expectations, 7 (3), 221 - 234.

Burkhardt, H.; Wehling, M.; Gladisch, R. (2007). Prävention unerwünschter Arzneimittelwirkungen bei älteren Patienten. Zeitschrift für Gerontologie und Geriatrie, 40 (4), $241-254$.

Busse, R.; Blümel, M.; Scheller-Kreinsen, D.; Zentner, A. (2010). Tackling chronic disease in Europe: Strategies, interventions, and challenges. Copenhagen: WHO Regional Office for Europe on behalf of European Observatory on Health Systems and Policies.

Chodosh, J.; Morton, S. C.; Mojica, W.; Maglione, M.; Suttorp, M. J.; Hilton, L.; Rhodes, S.; Shekelle, P. (2005). Meta-analysis: chronic disease selfmanagement programs for older adults. Annals of Internal Medicine, 143 (6), 427 - 438.

Coca, V.; Nink, K. (2010). Arzneimittelverordnungen nach Alter und Geschlecht. In: Schwabe, U.; Paffrath, D. (Hrsg.) Arzneiverordnungs-Report 2010. Berlin: Springer, 933 - 946.

Cooper, R. J.; Bissell, P.; Ward, P.; Murphy, E.; Anderson, C.; Avery, T.; James, V.; Lymn, J.; Guillaume, L.; Hutchinson, A.; Ratcliffe, J. (2011). Further challenges to medical dominance? The case of nurse and pharmacist supplementary pre- 
scribing. Health, online first DOI:10.1177/1363459310364159 hea.sagepub.com [18.11.2011].

Coulter, A.; Ellins, J. (2007). Effectiveness of strategies for informing, educating, and involving patients. British Medical Journal, 335, 24-27.

de Silva, D. (2011). Evidence: Helping people help themselves. A review of the evidence considering whether it is worthwhile to support selfmanagement. London: Health Foundation.

di Luzio, G. (2008). Medical dominance and strategic action: the fields of nursing and psychotherapy in the German health care system. Sociology of Health \& Illness, 30 (7), $1022-1038$.

Döhler, M. (1997). Die Regulierung von Professionsgrenzen. Struktur und Entwicklungsdynamik von Gesundheitsberufen im internationalen Vergleich. Frankfurt/Main: Campus.

Donabedian, A. (1992). Quality assurance in health care. Consumers role. Quality of Health Care, 1 (4), $247-251$.

Dybowski, S. (2005). «Soweit nicht anders verordnet ...»- HIV-positive Frauen im Spannungsfeld zwischen Compliance und Lebensgestaltung. Frankfurt/Main: Mabuse.

Gaidys, U. (2011). Qualität braucht Kompetenz und Verantwortung Herausforderungen und Perspektiven einer Advanced Nursing Practice für die Gesundheitsversorgung aus pflegewissenschaftlicher Sicht. Pflege, 24 (1), 15-20.

Garms-Homolová, V.; Schaeffer, D. (Hrsg.) (1998). Medizin und Pflege. Kooperation in der ambulanten Versorgung. Wiesbaden: Ullstein.

Gereoni, V.; Schönermark, M. P.; Hagen, A. (2007). Interventionen zur Verbesserung der Compliance bzw. Adherence in der Arzneimitteltherapie mit Hinblick auf den Therapieerfolg. Köln: DIMDI.
Geuter, G.; Müller-Mundt, G.; Panhorst, H. (2009): Entwicklung eines Qualifikationsprogramms in der ambulanten Pflege als Beitrag zur Erweiterung der Kompetenz von Pflegenden zur Selbstmanagementförderung. In: Walkenhorst, U.; Nauerth, A.; Bergmann-Tyacke, I.; Marzinzik, K. (Hrsg.). Kompetenzentwicklung im Gesundheitsund Sozialbereich. Bielefeld: UniversitätsVerlagWebler, 157 - 168.

Glaeske, G. (2010). Kosten senken an oder mit Arzneimitteln. Für mehr Rationalität und Effizienz in der Therapie mit Medikamenten. Der Internist, 51 (8), 1057 - 1063.

Greenhalgh, T. (2009). Patient and public involvement in chronic illness: beyond the expert patient. British Medical Journal, 338:b49, doi: 10.1136/bmj.b49 [18.11.2011].

Haslbeck, J. (2007). Bewältigung komplexer Medikamentenregime bei chronischen Erkrankungen - Herausforderungen aus Sicht chronisch Kranker (Veröffentlichungsreihe des Instituts für Pflegewissenschaft an der Universität Bielefeld, P07-136). Bielefeld: IPW. http://www.uni-bielefeld.de/gesundhw/ag6/downloads/ipw-136. pdf [18.11.2011].

Haslbeck, J. (2010). Medikamente und chronische Krankheit. Bern: Hans Huber.

Haslbeck, J.; Schaeffer, D. (2007). Selbstmanagementförderung bei chronischer Krankheit: Geschichte, Konzept und Herausforderungen. Pflege, 20 (2), $82-92$.

Haslbeck, J.; Schaeffer, D .(2009). Routines in medication management: the perspective of people with chronic conditions. Chronic Illness, 5 (3), $184-196$.

Haynes, R. B.; Taylor, D. W.; Sackett, D. L. (Eds.) (1979). Compliance in health care. Baltimore: Johns Hopkins University Press.
Haynes, R. B.; McDonald, H. P.; Garg, A. X.; Montague, P. (2005). Interventions for helping patients to follow prescriptions for medications (Cochrane Review). Cochrane Libary 3. Oxford: Update Software.

Isfort, M.; Weidner, F.; Neuhaus, A.; Kraus, S.; Köster, V. H.; Gehlen, D. (2010). Pflege-Thermometer 2009. Eine bundesweite Befragung von Pflegekräften zur Situation der Pflege und Patientenversorgung im Krankenhaus. Köln: Deutsches Institut für angewandte Pflegeforschung e.V. (dip), Köln. http:// www.dip.de [18.11.2011]

Kranich, C. (2004). Patientenkompetenz. Was müssen Patienten wissen und können? Bundesgesundheitsblatt - Gesundheitsforschung - Gesundheitsschutz, 47 (10), 950 - 956.

Koerfer, A.; Köhle, K.; Obliers, R. (1994). Zur Evaluation von Arzt-Patienten-Kommunikation - Perspektiven einer angewandten Diskursethik in der Medizin. In: Redder, A.; Wiese, I. (Hrsg.). Medizinische Kommunikation: Diskurspraxis, Diskursethik, Diskursanalyse. Opladen: Westdeutscher Verlag, $55-93$.

KrPflG (2003). Gesetz über die Berufe in der Krankenpflege und zur Änderung anderer Gesetze vom 16. Juli 2003. Bundesgesetzblatt (BGBl.) 2003, Teil I (36), 1442 - 1458.

Kuhlmey, A.; Höppner, K.; Schaeffer, D. (2011). Neue Aufgabenzuschnitte, Arbeitsteilungen und Kooperationsformen. In: Schaeffer, D.; Wingenfeld, K. (Hrsg.). Handbuch Pflegewissenschaft (Neuausgabe). Weinheim: Juventa, 661 - 679.

Loh, A.; Simon, D.; Bieber, C.; Eich, W.; Härter, M. (2007). Patient and citizen participation in Germany current state and future perspectives. Zeitschrift für Ärztliche Fortbildung und Qualität im Gesundheitswesen, 101 (4), 229-235. 
Lorig, K.; Holman, H. R. (2003). Selfmanagement education: History, definition, outcomes, and mechanisms. Annals of Behavioural Medicine, 26 (1), $1-7$.

Ludwig, A. (2005). Herausforderungen komplexer Medikamentenregime bei chronischen Erkrankungen Eine Literaturanalyse der Compliance- und Adhärenzdebatte (Veröffentlichungsreihe des Instituts für Pflegewissenschaft an der Universität Bielefeld, P05-130). Bielefeld: IPW. http://www.uni-bielefeld.de/ gesundhw/ag6/downloads/ipw130.pdf [18.11.2011].

McWilliam, C. L. (2009). Patients, persons or partners? Involving those with chronic disease in their care. Chronic Illness, 5 (4), 277 - 292.

Meuser, M.; Nagel, U. (2005). ExpertInneninterviews - vielfach erprobt, wenig bedacht. In: Bogner, A.; Littig, B.; Menz, W. (Hrsg.). Das Experteninterview. Wiesbaden: VS Verlag für Sozialwissenschaften, 71-94.

Müller-Mundt, G. (2011). Patientenedukation als Aufgabe der Pflege. In: Schaeffer, D.; Wingenfeld, K. (Hrsg.). Handbuch Pflegewissenschaft. Weinheim:Juventa, $705-726$

Müller-Mundt, G.; Schaeffer, D. (2011). Bewältigung komplexer Medikamentenregime bei chronischer Krankheit im Alter. Förderung des Selbstmanagements als Aufgabe der Pflege. Zeitschrift für Gerontologie und Geriatrie, 44 (1), 6 - 12.

Müller-Mundt, G.; Haslbeck, J.; Schaeffer; D. (2008). Bewältigung komplexer Medikamentenregime. Erfordernisse der Selbstmanagementunterstützung. In: Schaeffer, D.; Behrens, J.; Görres, S. (Hrsg.). Optimierung und Evidenzbasierung pflegerischen Handelns. Weinheim: Juventa, $30-55$.

Petermann, F. (Hrsg.) (1998). Compliance und Selbstmanagement. Göttingen: Hogrefe.
Pittrow, D.; Krappweis, J.; Kirch, W. (2002). Arzneimittelanwendung bei Alten- und Pflegeheimbewohnern im Vergleich zu Patienten in ambulanter Pflege bzw. ohne Pflegebedarf. Deutsche medizinische Wochenschrift, 127 (39), 1995 - 2000.

Pols, R. G.; Battersby, M. W.; ReganSmith, M.; Markwick, M. J.; Lawrence, J.; Auret, K.; Carter, J.; Cole, A.; Disler, D.; Hassed, C.; McGuiness, C. Nguyen, H. (2009). Chronic condition self-management support: proposed competencies for medical students. Chronic Illness, 5 (1), 7 - 14.

Robinson, J. H.; Callister, L. C.; Berry, J. A; Dearing , K. A. (2008). Patientcentered care and adherence: Definitions and applications to improve outcomes. Journal of the American Academy of Nurse Practitioners, 20 (12), 600 - 607.

RPSGB (1997). From compliance to concordance. London: Royal Pharmaceutical Society of Great Britain.

Schaeffer, D. (2004). Der Patient als Nutzer. Bern: Hans Huber.

Schaeffer, D. (2008). Patientenorientierte Versorgungskonzepte zur Bewältigung chronischer Krankheit. In: Schaeffer, D.; Behrens, J.; Görres, S. (Hrsg.). Optimierung und Evidenzbasierung pflegerischen Handelns. Weinheim: Juventa, $13-29$.

Schaeffer, D. (Hrsg.) (2009). Bewältigung chronischer Krankheit im Lebenslauf. Bern: Hans Huber.

Schaeffer, D.; Dewe, U. (2006). Zur Interventionslogik von Beratung in Differenz zu Information, Aufklärung und Therapie. In: Schaeffer, D.; Schmidt-Kaehler, S. (Hrsg.). Lehrbuch Patientenberatung. Bern Hans Huber, 127 - 152.

Schaeffer, D.; Moers, M. (2011). Bewältigung chronischer Krankheiten Herausforderungen für die Pflege. In: Schaeffer, D.; Wingenfeld, K.
(Hrsg.). Handbuch Pflegewissenschaft. Weinheim:Juventa, 329-363. Schaeffer, D.; Schmidt-Kaehler, S. (Hrsg.) (2006). Lehrbuch Patientenberatung. Bern: Hans Huber.

Schaeffer, D.; Müller-Mundt, G.; Haslbeck, J. (2007). Bewältigung komplexer Medikamentenregime bei chronischer Krankheit aus der Sicht der Gesundheitsprofessionen (Veröffentlichungsreihe des Instituts für Pflegewissenschaft an der Universität Bielefeld, P07-134). IPW: Bielefeld. http://www.unibielefeld.de/gesundhw/ag6/downloads/ipw-134.pdf [18.11.2011].

Schober, M.; Affara, F. (2008). Advanced nursing practice (ANP). Bern: Hans Huber.

Schwabe, U.; Paffrath, D. (Hrsg.) (2010). Arzneiverordnungs-Report 2010 Berlin: Springer.

Shaw, A.; De Lusignan, S.; Rowlands, G. (2005). Do primary care professionals work as a team: A qualitative study. Journal of Interprofessional Care, 19 (4), 396- 405.

Stollberg, G. (2008). Kunden der Medizin? Der Mythos vom mündigen Patienten. In: Saake, I.; Vogd, W. (Hrsg.). Moderne Mythen der Medizin. Wiesbaden: VS Verlag für Sozialwissenschaften, 345-362.

Strauss, A. (2007). Grundlagen qualitativer Sozialforschung. München Fink.

Strauss, A. L.; Corbin, J.; Fagerhaugh, S.; Glaser, B.; Maines, D. R.; Suczek, B.; Wiener, C. L. (1984). Chronic illness and the quality of life. Saint Louis: Mosby

SVR (2005). Koordination und Qualität im Gesundheitswesen. Gutachten 2005. Berlin: SVR. http://dipbt. bundestag.de/dip21/btd/16/063/ 1606339.pdf [18.11.2011].

SVR (2007). Kooperation und Verantwortung. Voraussetzungen einer zielorientierten Versorgung. Gutachten 2007. Berlin: SVR. http:// 
dip21.bundestag.de/dip21/btd/16/ 137/1613770.pdf [18.11.2011].

SVR (2009). Koordination und Integration - Gesundheitsversorgung in einer Gesellschaft des längeren Lebens. Sondergutachten 2009. Berlin: SVR. http://dip21.bundestag.de/dip21/btd/15/056/1505670. pdf [18.11.2011].

Thürmann, P. A.; Werner, U.; Hanke, F.; Schmiedl, S.; Drewelow, B.; Hippius, M.; Reimann, I. R., Siegmund, W.: Hasford, J. (2007). Arzneimittelrisiken bei hochbetagten Patienten: Ergebnisse deutscher Studien. In: BÄK (Hrsg.). Fortschritt und Fortbildung in der Medizin (Band 31). Köln: Deutscher Ärzte Verlag, 216-224.

van Dulmen, S.; Sluijs, E.; van Dijk, L.; de Ridder, D.; Herdink, R.; Bensing, J. (2007). Patient Adherence to Medical Treatment: A Review of Reviews. BMC Health Services 7: 55, http: / / www.biomedcentral. com/1472-6963/755 [18.11.2011].

van Mil, J. W. F.; Schulz, M.; Tromp, T. F. J. D. (2004). Pharmaceutical care:
European developments in concepts, implementation, teaching, and research: a review. Pharmacy World and Science, 26 (6), $303-311$.

Vermeire, E.; Hearnshaw, H.; van Royen, P.; Denekens, J. (2001). Patient adherence to treatment: three decades of research. A comprehensive review. Journal of Clinical Pharmacy and Therapeutics, 26 (5), 331 - 342.

von Uexküll, T.; Wesiak, T. (1990). Theorie der Humanmedizin. Grundlagen ärztlichen Handelns. München: Urban \& Schwarzenberg.

WHO (2003). Adherence to long-term therapies. Evidence in action. Genf: WHO.

WHO (2005). Preparing a health care workforce for the $21^{\text {st }}$ century. The challenge of chronic conditions. Genf: WHO.

Wingenfeld, K. (2011). Pflegebedarf, Pflegebedürftigkeit und pflegerische Leistungen. In: Schaeffer, D.; Wingenfeld, K. (Hrsg.). Handbuch Pflegewissenschaft. Weinheim: Juventa, $236-290$.
Winter, M. H. J. (2008). Anforderungsveränderung an die Qualifizierung der Gesundheitsberufe. In: Kuhlmey. A.; Schaeffer, D. (Hrsg.). Alter, Gesundheit und Krankheit. Bern: Hans Huber, 412 - 419.

Wu, T.-Y.; Jen, M.-H.; Bottle, A.; Molokhia, M.; Aylin, P.; Bell, D.; Majeed, A. (2010). Ten-year trends in hospital admissions for adverse drug reactions in England 1999-2009. Journal of the Royal Society of Medicine, 103 (6), 239 - 250.

\section{Korrespondenzadresse}

Prof. Dr. Doris Schaeffer

Fakultät für

Gesundheitswissenschaften, AG6

Versorgungsforschung/

Pflegewissenschaft

Universitätsstraße 25

DE-33615 Bielefeld

doris.schaeffer@uni-bielefeld.de 